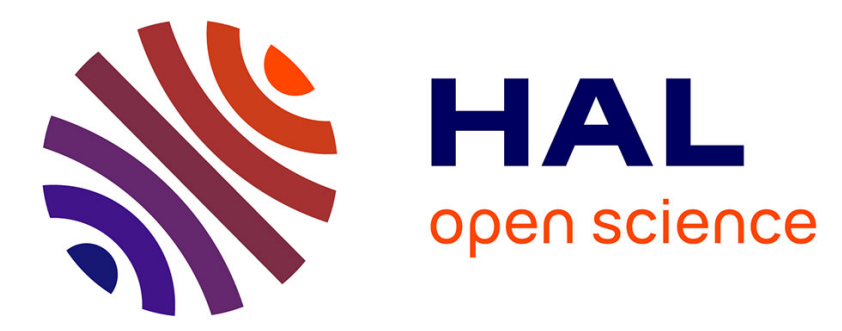

\title{
Dissipation Scalings in the Turbulent Boundary Layer at Moderate $\operatorname{Re} \theta$
}

\author{
Martin Obligado, C. Brun, J. Silvestrini, E. Schettini
}

\section{To cite this version:}

Martin Obligado, C. Brun, J. Silvestrini, E. Schettini. Dissipation Scalings in the Turbulent Boundary Layer at Moderate Re $\theta$. Flow, Turbulence and Combustion, 2021, 10.1007/s10494-021-00270-1 . hal03241514

\section{HAL Id: hal-03241514 \\ https://hal.science/hal-03241514}

Submitted on 30 Sep 2021

HAL is a multi-disciplinary open access archive for the deposit and dissemination of scientific research documents, whether they are published or not. The documents may come from teaching and research institutions in France or abroad, or from public or private research centers.
L'archive ouverte pluridisciplinaire HAL, est destinée au dépôt et à la diffusion de documents scientifiques de niveau recherche, publiés ou non, émanant des établissements d'enseignement et de recherche français ou étrangers, des laboratoires publics ou privés. 


\title{
Dissipation Scalings in the Turbulent Boundary Layer at Moderate $\operatorname{Re}_{\theta}$
}

\author{
M. Obligado ${ }^{1}$ (D) . C. Brun ${ }^{1}$ (D) J. H. Silvestrini ${ }^{2}$ (D) E. B. C. Schettini ${ }^{3}$ (D)
}

\begin{abstract}
We present an experimental and numerical study of the turbulent boundary layer at $1200<R e_{\theta}<3400$. We propose a combined approach that involves the use of a multi-hole pitot probe, hot-wire anemometry and direct numerical simulations, that allows to characterise the isotropy and the energy dissipation scalings of the flow in the outer layer, in the range $0.4<y / \delta<0.75$, with $y$ the vertical coordinate and $\delta$ the boundary layer thickness. We confirm previous results from the literature that show that for low values of $\operatorname{Re}_{\theta}$ (in our case $R e_{\theta}<2500$ ), on the outer layer the dissipation constant $C_{\varepsilon}$ is Reynolds number dependent, following a $R e_{\lambda}^{-1}$ law. This dependency seems to be robust, as the value of $C_{\varepsilon}$ collapses for previously reported direct numerical simulations and experiments with different incoming velocities. We also show that, while large scales of the flow are strongly anisotropic on the outer layer, the turbulence energy dissipation rate and the dissipation constant can still be characterised assuming local isotropy and homogeneity.
\end{abstract}

Keywords Turbulent boundary layer $\cdot$ Turbulence energy cascade $\cdot$ Dissipation constant . Experimental fluid dynamics

\section{Introduction}

The turbulent boundary layer (TBL) is a flow that has received widespread attention from the turbulence community over the last decades, mainly due to its fundamental role in aeronautics, drag reduction and environmental applications (Smits et al. 2011; Marusic 2009). It is a canonical flow that combines both fundamental physics and geophysical and industrial applications. Its simple geometry makes it particularly suitable for its study via experiments in wind tunnels and computational fluid dynamics.

M. Obligado

Martin.Obligado@univ-grenoble-alpes.fr

1 Université Grenoble Alpes, CNRS, Grenoble-INP, LEGI, F-38000 Grenoble, France

2 Escola Politécnica, Pontifícia Universidade Católica do Rio Grande do Sul, Av. Ipiranga 6681, 90619-900 Porto Alegre, RS, Brazil

3 Instituto de Pesquisas Hidráulicas (IPH), Universidade Federal do Rio Grande do Sul, Porto Alegre, RS, Brazil 
Despite this intense activity, many open questions remain, as evidenced by the recent discovery of the outer peak in the mean squared fluctuating streamwise velocity and the new theoretical models developed in consequence (Vassilicos et al. 2015; Hultmark et al. 2012). Nedić et al. have also recently reported the presence of non-equilibrium turbulence (Nedić et al. 2017) in both experimental and direct numerical simulation (DNS) data.

The non-equilibrium energy cascade has been reported in several flows during the last decade: forced periodic turbulence and decaying periodic turbulence (Goto and Vassili$\cos 2016 \mathrm{a}$, b; Valente et al. 2014), various types of grid-generated turbulence (Vassilicos 2015; Mora et al. 2019; Hearst and Lavoie 2016; Nagata et al. 2017), turbulent free-shear flows (Vassilicos 2015; Obligado et al. 2016; Cafiero and Vassilicos 2019), and other shear flows (Nedić and Tavoularis 2016; Takamure et al. 2019). The Richardson-Kolmogorov cascade predicts that the turbulent dissipation rate evolves as $\varepsilon=C_{\varepsilon} u^{\prime 3} / L$, with $C_{\varepsilon}$ being a constant, $L$ the longitudinal integral length scale, and $u^{\prime}$ the root mean square (rms) of the longitudinal velocity fluctuations. On the other hand, within the non-equilibrium cascade $C_{\varepsilon}$ is not constant, but instead it goes as $C_{\varepsilon} \sim R e_{G} / R e_{L}$, for large enough $R e_{\lambda}$ (defined as $\operatorname{Re}_{\lambda}=\lambda u^{\prime} / v$, with $\lambda$ the Taylor microscale and $v$ the kinematic viscosity of the flow). $R e_{G}$ is a global Reynolds number that depends on the inlet conditions (not present in the Richardson-Kolmogorov cascade) and $R e_{L}$ a local, streamwise position dependent parameter (usually defined with the integral lengthscale and a local rms velocity). It can also be shown that an equivalent expression for $C_{\varepsilon}$ is $C_{\varepsilon} \sim \sqrt{R e_{G}} / R e_{\lambda}$ (Vassilicos 2015).

The modelling of $C_{\varepsilon}$ via the Richardson-Kolmogorov closure makes part implicitly and explicitly in theoretical and numerical modelling of turbulent flows (Vassilicos 2015; Pope 2001; Lesieur 2012). For instance, it is used to estimate the number of degrees of freedom of a turbulent flow, eddy viscosity models, large-eddy simulations, etc... its role is so preeminent that some authors have defined the scaling for $\varepsilon$ as the zeroth-law of turbulence (Lumley 1992). In fact, it can be deduced from the Kolmogorov's 4/5 law (Monin and Yaglom 2013), and therefore it is closely linked to the Richardson-Kolmogorov modelling of the direct energy cascade in homogeneous isotropic turbulence. The presence of a different scaling for $\varepsilon$ therefore invalidates some of the assumptions from the standard model stated above. Nevertheless, the dissipation constant is one of the hardest turbulence parameters to measure experimentally, as it requires exceptional spatial and/or temporal (when it is obtained via the Taylor hypothesis) resolution, and still remains beyond our current experimental capabilities for very large Reynolds numbers.

While the energy cascade in the TBL is a very complex process (Castillo and George 2001; George and Castillo 1997) that depends on many factors as the distance from the wall, the local value of $R e_{\lambda}$, among others, recent works have focused on the outer region of spatially evolving turbulent boundary layers (Nedić et al. 2017; Kamruzzaman et al. 2018). The turbulent flow within this region is developed, and therefore can be approximated, to some extent, as homogeneous and isotropic on the small scales (we will discuss this point in detail in the next section). A recent work (Nedić et al. 2017) found the presence of anomalous dissipation scalings on the TBL for $R e_{\theta}<10^{4}$, with $R e_{\theta}=U_{\infty} \theta / \nu$, a Reynolds number based on the freestream velocity $U_{\infty}$ and the momentum thickness $\theta$ (defined on the next section). They studied DNS and hot-wire anemometry (HW) in the outer region of a spatially evolving turbulent boundary layer in the range $0.3<y / \delta<0.7$, with $y$ the vertical coordinate and $\delta$ the boundary layer thickness. While there is no consensus on the exact value of $y / \delta$ for which the outer layer begins, the range explored in this work corresponds to values generally accepted to be part of such region (Phillips and Ratnanather 1990; Sreenivasan 1989). Whereas recent works confirm these findings (Liu 
et al. 2021), there still remains many open questions about the structure and properties of the energy cascade that we will address in the present work.

First, we compare DNS of the turbulent boundary layer with experimental data for $R e_{\theta}$ in the range $1000<R e_{\theta}<3500$, where $C_{\varepsilon}$ is expected not to be constant but to follow a $R e_{\lambda}^{-1}$ power law. Experiments are performed with both a single hot wire (HW) and a Cobra probe. The first device allows to resolve small-scale turbulence quantities in $1 \mathrm{D}$, such as $\varepsilon$ while the second can resolve the mean and rms values of the velocity vector in $3 \mathrm{D}$ within its limited spatial and temporal resolution, discussed in detail in the next section. To the authors best knowledge, no such complementary study involving DNS and HW and Cobra probes has been made before. We remark that the Cobra probe is not adapted for a detailed study of the TBL, as its low spatial and moderate temporal resolution are insufficient for resolving the small-scale properties of the flow. We therefore have used it only to characterise the large-scale anisotropy of the flow.

We can therefore address, using DNS data (that covers, in our case, the range $R e_{\theta}<1400$ ), which is the vertical range where $\varepsilon$ can be approximated using the local homogeneity and isotropy assumptions for the flow velocity field. This assumption is needed to deduce the value of $C_{\varepsilon}$ experimentally with a single hot wire. The relevance of this problem has already been raised in previous works in the TBL (Kamruzzaman et al. 2018; Pumir et al. 2016), as an incorrect estimation of the turbulent dissipation rate could affect the conclusions obtained in terms of the energy cascade and the quantification of $C_{\varepsilon}$, needed for turbulence modelling. After this point is clarified, the HW and the Cobra probes can be used to study the dissipation scalings for larger values of $R e_{\theta}$. Our combined approach allows to quantify the large scale $3 \mathrm{D}$ properties of the flow (via the mean and rms values of the velocity vector obtained with the Cobra probe) and the small scale ones from the streamwise components (as we discuss in the next section, the HW resolved $\varepsilon$ via the dissipation spectra and therefore could be used to quantify all small-scale single point parameters such as the Kolmogorov lengthscale $\left.\eta, \lambda, \operatorname{Re}_{\lambda}, \ldots\right)$.

Furthermore, the comparison between HW, Cobra and DNS allows to address other issues on the characterisation of the energy cascade via the dissipation scalings. In fact, it has been found in other inhomogeneous/anisotropic flows (like the axisymmetric turbulent wake (Dairay et al. 2015; Obligado et al. 2016) and the planar jet (Cafiero and Vassili$\cos 2019)$ ) that a better estimation of $C_{\varepsilon}$ is obtained using the kinetic energy instead of $u^{\prime}$ : $C_{\varepsilon}=\varepsilon L / K^{3 / 2}$. Our experimental setup allows to compare both definitions. Therefore, we propose a study that can characterise the energy cascade considering the anisotropic nature of both large and small scales of the flow.

We also discuss the role of $R e_{G}$ in the non-equilibrium energy cascade in the TBL. This is not trivial to explore, as it is even unclear how to define an inlet Reynolds number on this flow. It is usually defined via a characteristic length and velocity at the inlet of the flow (for example, the freestream velocity and frontal characteristic length for a wake). While the definition of an inlet length-scale for the TBL is unclear, $R e_{G}$ should be a linear function of $U_{\infty}$. We have then performed measurements at several different incoming freestream velocities but at fixed streamwise positions. To the authors knowledge, no previous study of the dependency of $C_{\varepsilon}$ with $U_{\infty}$ has been reported before in this flow.

To summarise, we present a numerical/experimental approach, where DNS, 1D HW and 3D Cobra techniques are complemented to characterise the TBL at moderate values of $R e_{\theta}\left(R e_{\theta}<3400\right.$ and $\left.50<R e_{\lambda}<140\right)$. While the characteristics of the wind tunnel used in this work do not allow us to explore larger values of this parameter, this is a range frequently tested in several numerical and experimental studies. Within this work, we will first show that such complementary approach can be used to obtain all one point statistics of 
the turbulent flow (via the Taylor hypothesis), including $C_{\varepsilon}, \eta, K, L, \ldots$ we will then use the DNS results to show that $\varepsilon$ can be approximated using the standard formulae that assume small scales remain homogeneous and isotropic (and therefore can be estimated with a single $\mathrm{HW}$ ). We will then use our data to evaluate the properties of the energy cascade (as modelled by $C_{\varepsilon}$ ), and we will confirm the trends from (Nedić et al. 2017) that suggest the presence of a non-equilibrium cascade. Furthermore, we will study the role of $\operatorname{Re}_{G}$ on it. Finally, we will confirm by means of the Cobra probe measurements, that considering the large-scale anisotropy of the flow do not modify these conclusions.

\section{Numerical methods}

The numerical results in this article are based on a turbulent boundary layer computed using DNS in a computational domain of dimensions $360 \delta_{0} \times 40 \delta_{0} \times 15 \delta_{0}$ (where $\delta_{0}$ is the inlet boundary layer thickness) with $3073 \times 513 \times 256$ mesh nodes. The simulations are performed with the high-order flow solver Incompact $3 d$ (available at www.incom pact3d.com), which is based on 6th order compact finite difference schemes to discrete the incompressible Navier-Stokes equations on a Cartesian mesh stretched in the wall normal direction and a 3rd order Adams-Bashforth scheme for time advancement. The incompressibility condition is treated with a pseudo-spectral approach to solve the Poisson equation for the pressure. Additional information about the numerical code can be found in Laizet and Lamballais (2009); Li and Laizet (2011). For the inlet boundary condition a Blasius boundary layer is prescribed with local Reynolds number equal to $\operatorname{Re}_{\delta_{0}}=2000$ corresponding to $R e_{\theta}=270$. A by-pass procedure using a tripping method proposed in Schlatter and Örlü (2012) is used to reach turbulent conditions. At the outlet, where the Reynolds number reaches $R e_{\theta}=1640$, an advection equation is solved for the boundary condition. Periodic boundary conditions are imposed in the spanwise direction, while classical no-slip and free-slip boundary conditions are set, respectively, at the wall and top of the computational domain. Typical values for the spatial and time resolution in wall units for $R e_{\theta}=1400$ ( or $R e_{\tau}=452$, based on the skin friction velocity $u_{\tau}$ and the boundary layer thickness $\delta$ ) are $\Delta x^{+}=10.4, \Delta y^{+}=0.8$ (at the wall, where $y^{+}=y u_{\tau} / v$ ), $\Delta z^{+}=5.2$ and $\Delta t^{+}=0.028$ (corresponding to $\Delta t=0.007 \delta_{0} / U_{\infty}$ ). The domain configuration and numerical parameters used for this article are close to those defined in Diaz-Daniel et al. (2017) and the results have been validated with the KTH's database (Schlatter and Örlü 2010).

In the present DNS the Reynolds number reached at the outlet of the domain is in the order of the smaller value of $R e_{\theta}$ reported in experiments (see next section). The relevance of this part of the study is twofold. First, it allows to study the influence of small-scale anisotropy and inhomogeneity on the estimation of $C_{\varepsilon}$ (not accessible with HW and Cobra probe measurements). It will also be used to validate the range of wall distances in which HW and Cobra experimental results are valid, as the relatively small values of $\operatorname{Re}_{\theta}$ and $\delta$ generated ( $\delta$ is below $5 \mathrm{~cm}$ for all cases) imply that the log-law layer is extremely thin (of a few $\mathrm{mm}$ ) and could be affected by the HW and Cobra probe and their holders. We can therefore verify, by comparing DNS, Cobra and HW profiles, that the experimental data remains valid in the outer layer. As we detail in the next section, we also use the DNS scalings to estimate $u_{\tau}$ for our experiments (via a direct measurement of $R e_{\theta}$ ). These parameters will be used to normalise different quantities with wall units and match DNS and experimental results. 

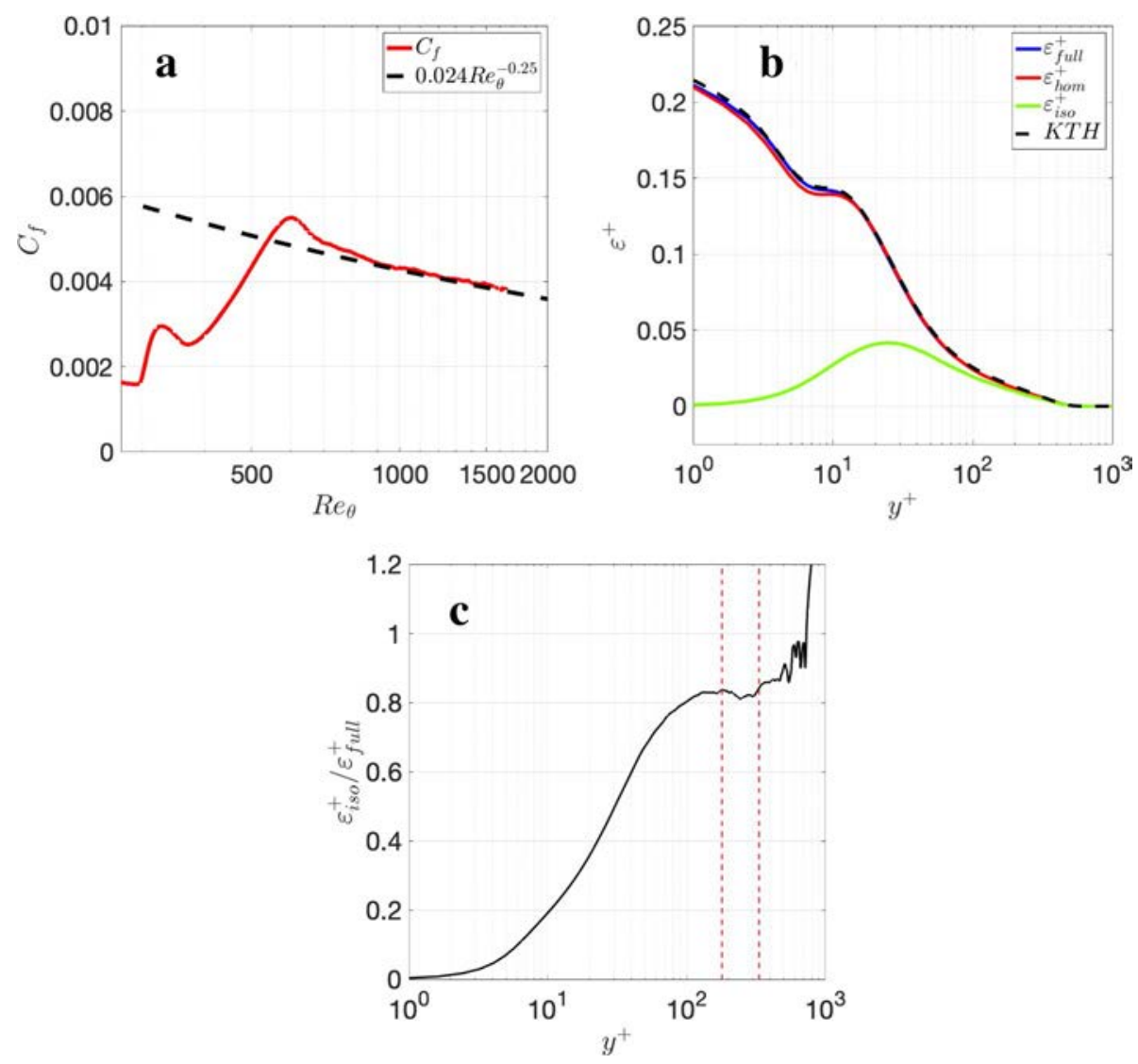

Fig. 1 Friction coefficient $C_{f}$ as a function of $R e_{\theta}$ a. The black dashed line correspond to the fit from the DNS reported in Schlatter and Örlü (2010). Different definitions of $\varepsilon$, as defined in the text b. The black dashed line corresponds to the estimation of $\varepsilon_{\text {full }}$ from Schlatter and Örlü (2010). Ratio between $\varepsilon_{\text {iso }}$ and $\varepsilon_{f u l l}$ c. The vertical lines correspond to $y / \delta=0.4$ and $y / \delta=0.75$. Figures $\mathbf{b}$ and $\mathbf{c}$ correspond to $\operatorname{Re}_{\theta}=1400$

Figure 1a shows the evolution of the friction coefficient $C_{f}$ with $\operatorname{Re}_{\theta}$ obtained with the DNS. It can be observed that our DNS collapse very well with the fit proposed by Schlatter and Örlü (2010), $C_{f}=0.024 R e_{\theta}^{-1 / 4}$. On the other hand, Fig. 1b shows three different estimations for $\varepsilon$ as a function of the vertical coordinate $y$ (both parameters are normalised with wall units, and therefore $\varepsilon^{+}=\varepsilon v / u_{\tau}^{4}$ ). $\varepsilon$ is estimated following the definitions from Mi and Antonia (2010). The exact form of $\varepsilon$ is,

$$
\varepsilon_{f u l l}=v\left\langle\left(\frac{\partial u_{i}}{\partial x_{j}}+\frac{\partial u_{j}}{\partial x_{i}}\right) \frac{\partial u_{j}}{\partial x_{i}}\right\rangle .
$$

This expression, can be simplified using different assumptions. First, if the flow is assumed to be locally homogeneous, the expression can be rewritten as, 


$$
\begin{aligned}
\varepsilon_{\text {hom }} & =v\left\langle\left(\frac{\partial u}{\partial x}\right)^{2}\right\rangle+\left\langle\left(\frac{\partial u}{\partial y}\right)^{2}\right\rangle+\left\langle\left(\frac{\partial u}{\partial z}\right)^{2}\right\rangle+\left\langle\left(\frac{\partial v}{\partial x}\right)^{2}\right\rangle \\
& +\left\langle\left(\frac{\partial v}{\partial y}\right)^{2}\right\rangle+\left\langle\left(\frac{\partial v}{\partial z}\right)^{2}\right\rangle+\left\langle\left(\frac{\partial w}{\partial x}\right)^{2}\right\rangle+\left\langle\left(\frac{\partial w}{\partial y}\right)^{2}\right\rangle+\left\langle\left(\frac{\partial w}{\partial z}\right)^{2}\right\rangle,
\end{aligned}
$$

where $u, v$ and $w$ correspond to the streamwise ( $x$ coordinate), the wall normal $(y)$ and the lateral $(z)$ fluctuating velocities, respectively. Finally, for locally homogeneous isotropic turbulence, $\varepsilon$ takes the form,

$$
\varepsilon_{i s o}=15 v\left\langle\left(\frac{\partial u}{\partial x}\right)^{2}\right\rangle
$$

that can potentially be estimated experimentally using hot-wire anemometry. While this last expression is usually employed to obtain $\varepsilon$ for experimental data, it is not clear if it is valid or not, and if it is, in which regions of the TBL it can be employed. To cater this question, in Fig. $1 \mathrm{~b}$ can be observed that for $y^{+}$larger than 150, all three definitions give similar results. In particular, the ratio $\varepsilon_{i s o} / \varepsilon_{\text {full }}$ presents a plateau in the outer layer (with a value of $\sim 0.8$ in the range $0.4<y / \delta<0.75$, see Fig. 1c). The existence of such plateau validates the approach proposed by previous works (Nedić et al. 2017; Kamruzzaman et al. 2018). Nevertheless, it is remarkable that the difference between $\varepsilon_{i s o}$ and $\varepsilon_{f u l l}$ is larger than in other inhomogenous flows (see for instance (Dairay et al. 2015), where for an axisymmetric turbulent wake this difference remains below 6\%). These results, that suggest that anisotropy is still relevant at small scales, should be addressed in further DNS studies.

\section{Experimental Setup}

Experiments were conducted in the Lespinard wind tunnel at LEGI-UGA: a large wind tunnel with a measurement section of $4 \mathrm{~m}$ long and a square cross-section of $0.75 \times 0.75 \mathrm{~m}^{2}$. The TBL is generated with a PMMA wall of $3 \mathrm{~m}$ long, $720 \mathrm{~mm}$ wide and a thickness of $20 \mathrm{~mm}$. It was placed horizontally on the floor of the wind tunnel. A blunt finishing, with a parabolical profile was added to allow the laminar BL to develop and avoid BL detachment. A thin piece of rough tape, placed transversally to the inlet of the wall (immediately downstream the parabolic end) was used as a tripper. Nevertheless, we did not confirm that the transition occurred always at that point, so the streamwise origin of the turbulent boundary layer could have some dependency with the freestream velocity. For all cases, outside the TBL, the turbulent intensity (for the tunnel with the PMMA wall inserted) was below $0.2 \%$ and the pressure gradient negligible.

All HW measurements were made by means of a single hot wire, using a Dantec Dynamics 55P01 hot-wire probe, driven by a Dantec StreamLine CTA system. The Pt-W wires were $5 \mu \mathrm{m}$ in diameter, $3 \mathrm{~mm}$ long with a sensing length of $l_{w}=1.25 \mathrm{~mm}$. Acquisitions were made for $60 \mathrm{~s}$ at sampling frequencies between $20 \mathrm{kHz}$ and $30 \mathrm{kHz}$ (a low-pas filter was always active at $30 \mathrm{kHz}$ to counteract for aliasing). This correspond to boundary layer turnover times (defined via $T U_{\infty} / \delta$, with $\mathrm{T}$ the acquisition time), between $4.3 \times 10^{3}$ and $17 \times 10^{3}$. We performed some tests at longer acquisition times and higher sampling frequencies to discard the presence on any convergence problems. It was checked that for all the datasets where $C_{\varepsilon}$ results are reported we have at least $k \eta=\frac{2 \pi}{U} f \eta=1$ (with $U$ the 
local streamwise mean velocity, $k=2 \pi f / U$ is the respective wave number, $f$ the largest frequency resolved in our data (before the onset of noise) and $\eta$ the Kolmogorov lengthscale, $\left.\eta=\left(v^{3} / \varepsilon\right)^{1 / 4}\right)$. On the same range, the hot wire has a spatial resolution, $l_{w} / \eta$ between 4 (lower value of $U_{\infty}$ ) and 9.5 (higher value), as it can also be deduced from results reported later in Fig. 5. The HW was calibrated with a standard pitot tube, and both devices were placed at the inlet of the wind tunnel for each calibration. The pitot tube was then removed from the wind tunnel during measurements.

Large scale isotropy was quantified with a Cobra probe manufactured by TFI (Watkins et al. 2002), which is able to compute the three fluctuating velocities $(u, v, w)$ plus their mean $(U, V, W)$ and rms $\left(\left(u^{\prime}, v^{\prime}, w^{\prime}\right)\right.$, where $u^{\prime}$ actually correspond to the standard deviation of $u$ ) values, with a temporal resolution of $1250 \mathrm{~Hz}$. The acquisition time was set to $120 \mathrm{~s}$ at the maximum sampling frequency (and consequently between $8.6 \times 10^{3}$ and $34 \times 10^{3}$ boundary layer turnover times). We remark that the probe has a $4 \mathrm{~mm}^{2}$ sensitive area, and therefore is prone to have size effects. Their relevance will be further discussed in the next section, particularly as this area represent a characteristic length (of around $2 \mathrm{~mm}$ ) larger than $\eta$ and similar to $\lambda$ for our data. To the authors knowledge, this effect cannot be corrected, as it is a consequence from the spatial filtering due to the sensor head size.

Vertical profiles were measured at $x=2.25 \mathrm{~m}$, with $x$ the distance between the probe and the blunt end of the plate. Four different incoming velocities where tested: $U_{\infty}=3.5,6.2,9.7$ and $11.9 \mathrm{~m} / \mathrm{s}$. Another profile, at $x=2 \mathrm{~m}$ and $U_{\infty}=6.9 \mathrm{~m} / \mathrm{s}$ was also measured. For each $U_{\infty}$ and $x$-position, a vertical profile was performed between $y=1 \mathrm{~mm}$ and $y=100 \mathrm{~mm}$ that comprised 26 points. HW and Cobra measurements were used alternatively for the same vertical points and experimental conditions.

Table 1 shows the boundary layer parameters for all experimental conditions explored. The momentum thickness is defined as $\theta=\int_{0}^{\infty} \frac{u(y)}{U_{\infty}}\left(1-\frac{u(y)}{U_{\infty}}\right) d y$ and $\delta$ is the corresponding 99\% boundary layer thickness. The associated Reynolds number is $\operatorname{Re}_{\theta}=U_{\infty} \theta / \nu$ and the friction coefficient $C_{f}$ has been estimated using the experimental value of $\theta$ and the fit from Fig. 1a. The friction velocity becomes $u_{\tau}=\sqrt{\frac{1}{2} C_{f} U_{\infty}^{2}}$ (the reasons are detailed below).

Figures $2 \mathrm{a}$ and $\mathrm{b}$ show a comparison of the normalised mean streamwise velocity and its standard deviation, $U^{+}$and $u^{+}$(note that we define the latter as $u^{+}$and not $u^{\prime+}$, while it still corresponds to the rms value of the fluctuating velocity, respectively, while all velocities in wall units reported here have been normalised using $u_{\tau}$ ) between HW and DNS data. It can be observed that indeed, for $y^{+}>200$ the lower value of $U_{\infty}$ and the DNS (with a similar

Table 1 Experimental parameters. $x$ stands for the distance from the probe to the edge of the plate, $U_{\infty}$ is the inlet velocity, $\delta$ is defined as the corresponding $99 \%$ TBL thickness.

\begin{tabular}{rcllllllr}
\hline$x(m)$ & $U_{\infty}(\mathrm{m} / \mathrm{s})$ & $\delta(\mathrm{mm})$ & $\theta(\mathrm{mm})$ & $R e_{\theta}$ & $R e_{\delta}$ & $C_{f}$ & $u_{\tau}(\mathrm{m} / \mathrm{s})$ & $R e_{\tau}$ \\
\hline 2.25 & 3.5 & 49.2 & 5.30 & 1240 & 11500 & 0.0040 & 0.16 & 520 \\
2.25 & 6.2 & 49.2 & 5.40 & 2270 & 20000 & 0.0035 & 0.26 & 860 \\
2.25 & 9.7 & 43.8 & 4.40 & 2850 & 28000 & 0.0033 & 0.40 & 1150 \\
2.25 & 11.9 & 41.7 & 4.20 & 3370 & 33000 & 0.0032 & 0.48 & 1330 \\
2.00 & 6.9 & 39.9 & 4.20 & 1900 & 18000 & 0.0036 & 0.29 & 770 \\
\hline
\end{tabular}

The table also includes the momentum thickness $\theta$ and its associated Reynolds number is $\operatorname{Re}_{\theta}=U_{\infty} \theta / \nu$. The boundary layer thickness-based Reynolds number is defined as $\operatorname{Re}_{\delta}=U_{\infty} \delta / \nu$. $C_{f}$ and $u_{\tau}$, the friction coefficient and the skin friction velocity respectively, have been estimated from the DNS. The value of $R e_{\tau}$ is also included 

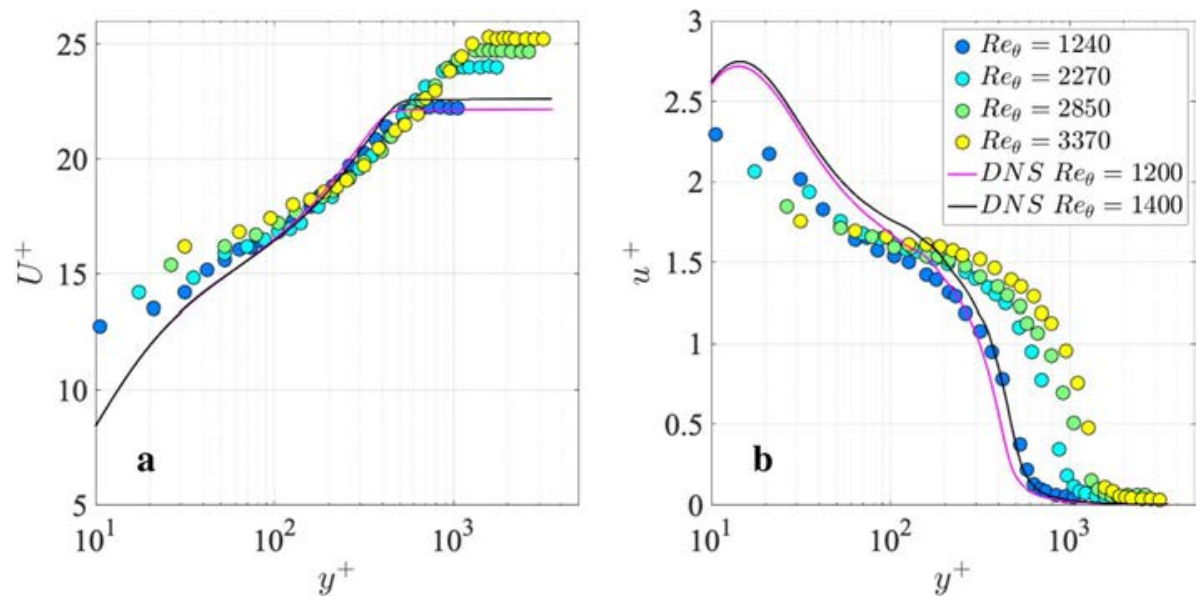

Fig. 2 Normalised mean (a) streamwise velocity and its standard deviation $\left(U^{+}\right.$and $u^{+}$, respectively) vs the vertical coordinate $y^{+}$(all in wall units) for HW measurements at $x=225 \mathrm{~cm}$ and DNS data

value of $R e_{\theta}$ ) are in good agreement, both for the mean and rms values of $u . y^{+}=200$ correspond, for our TBL, to $y<10 \mathrm{~mm}$, and the depart at lower $y^{+}$from the DNS of the TBL is probably caused by the bad resolution of the traverse system in the vertical direction (see below). Other cause could be an interference between the flow and the HW support close to the wall. Nevertheless, the range resolved in our dataset corresponds, for the worse case (lower $R e_{\theta}$ ) to $y / \delta>0.5$, and therefore the HW gets reliable measurements for almost the whole the range of interest for our study of $C_{\varepsilon}$ (higher values of $R e_{\theta}$ always fall within the range studied in Nedić et al. (2017)). Figure 2 also shows that the log-law region is too thin in our wind tunnel, and therefore the value of $u_{\tau}$ has not been resolved experimentally. We have nevertheless verified that $\theta$ is accurately estimated with the HW (with an error of $2 \%$ at most), and used its value to deduce $u_{\tau}$ from the fit of Fig. 1a.

We then compare the measurements from HW and Cobra probes (Fig. 3a). In this case, we expect to resolve only mean and rms quantities with the Cobra probe, that will be used to evaluate the kinetic energy on the computation of $C_{\varepsilon}$. In the figure it can be observed that it is indeed the case for our data: differences between both curves are below our velocity error, of $2 \%$, discussed below. Also, the freestream velocity for both cases may not be identical, as there may be a small variation in this parameter when setting the motors at a given power (caused by differences in the relative humidity, temperature and absolute pressure of the air). The figure does not include error bars, as they would mask the dependency of both curves with $y^{+}$. The absolute error for the $y$ coordinate position was of $0.5 \mathrm{~mm}$, and the temperature's is of $0.1^{\circ} \mathrm{C}$. While the error from the Cobra probe is below $0.2 \mathrm{~m} / \mathrm{s}$, we estimate the error from the HW in $0.1 \mathrm{~m} / \mathrm{s}$. This last value, has been chosen in a conservative way to consider variations of temperature with respect to the value on the calibration. While we monitored the temperature during measurements, we tolerated variations of up to $0.5^{\circ} \mathrm{C}$ between calibration and measurements. Otherwise, time-series from the Cobra and the HW are long enough to converge all statistics (at least $10^{3}$ integral time scales for a given time series, Fig. 3b). With this values, and assuming the error from DNS values is negligible compared to experiments, the absolute error of $C_{f}$ and normalised velocities is always below $1 \%$ and for $u_{\tau}$ below $3 \%$. The error for $y^{+}$remains below $4 \%$ for $y / \delta>0.3$. Finally, the error on $\theta$ and $\operatorname{Re}_{\theta}$ is always below $2 \%$. 

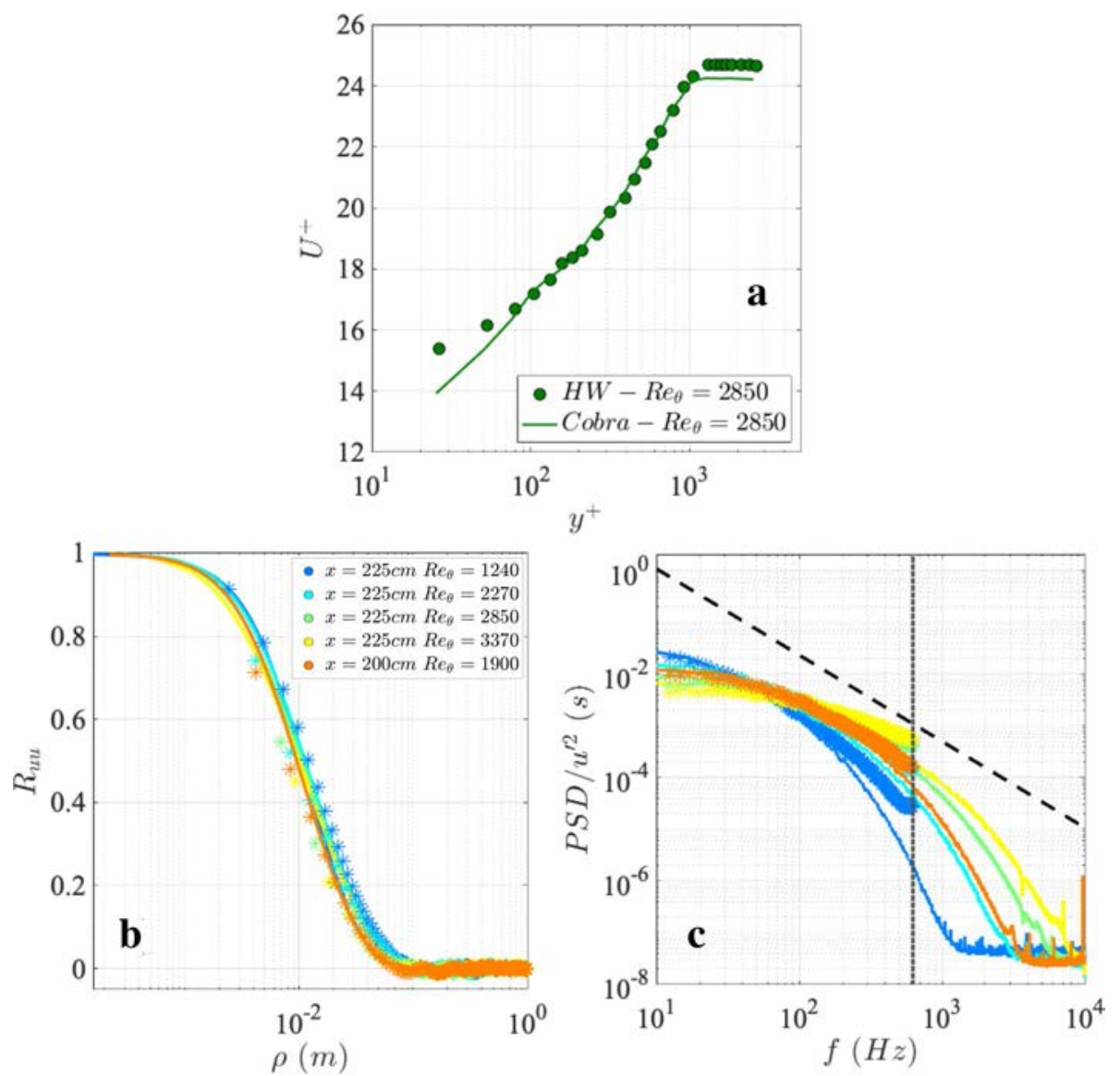

Fig. 3 Comparison of mean velocity profiles $U^{+}\left(y^{+}\right)$obtained with the Cobra probe and the HW at $x=225 \mathrm{~cm}$ and $\operatorname{Re}_{\theta}=2850$ (a). Normalised autocorrelation functions $R_{u u}(\rho)(\mathbf{b})$ and power spectral densities (c) at $y=30 \mathrm{~mm}$ (that correspond to values of $y / \delta$ between 0.6 and 0.75 ) obtained with the cobra probe (stars) and the HW (solid lines). The black dashed line corresponds to a $-5 / 3$ power law

Figure $3 \mathrm{c}$ shows the power spectral density for all $\operatorname{Re}_{\theta}$ at $y=30 \mathrm{~mm}$ for all values of $R e_{\theta}$, therefore always on the outer region of the TBL. It can be observed that the temporal power spectral density measured with both probes is very close for frequencies up to $100 \mathrm{~Hz}$ (a good agreement is also observed throughout the autocorrelations shown in Fig. 3b). This, added to the good agreement between the mean velocity profiles at $y^{+}>100$ implies that the Cobra probe can be used to complement HW data in terms on mean and rms values of the velocity vector. The presence and extent of a 5/3 power law in the power spectral densities is debatable, particularly for the lower values of $R_{\theta}$. Nevertheless, their shape is similar to those previously reported in the literature at similar values of $\operatorname{Re}_{\theta}$ (see for instance Solak and Laval 2018; Liu et al. 2021). This is a range studied by many numerical and experimental studies, and therefore it is important to address the properties of the dissipation scalings on it. Furthermore, the shape of power spectral densities still remains very similar to regular static grid spectra previously reported for similar values of $R e_{\lambda}$ (Antonia et al. 2014; Mora et al. 2019; Larssen and Devenport 2011). 


\section{Results}

Following the comparison between numerical and experimental data, Fig. $4 \mathrm{a}-\mathrm{c}$ show the rms of all fluctuating velocity components for Cobra and DNS (for $u^{+}$we also added the HW data). This figure confirms that these experimental quantities have good agreement to the DNS for $y^{+}>300$ (that gets increasingly better for larger $y^{+}$). While our DNS only resolves the smallest value of $R e_{\theta}$ from experiments, the trends of the vertical profiles with $y^{+}$are consistent with other DNS studies (Schlatter et al. 2010). Also, both the HWA (reported in Fig. 2b) and Cobra probe values for $y^{+}>300$ at $R e_{\theta}=3370$ present similar values to the DNS from Schlatter and Örlü (2010) at $R e_{\theta}=4064$.

Figure $4 \mathrm{~d}$ shows a comparison for only the Cobra and the DNS of the Reynolds stress $\left(u v^{+}\right.$, not needed to estimate the kinetic energy), that can be in principle be resolved with the multi-hole Pitot probe. While a good agreement is observed for $y^{+}>400$, this parameter shows a significantly worse collapse with the experimental data. In consequence, the values of the minima, that could have been used to determine $u_{\tau}$
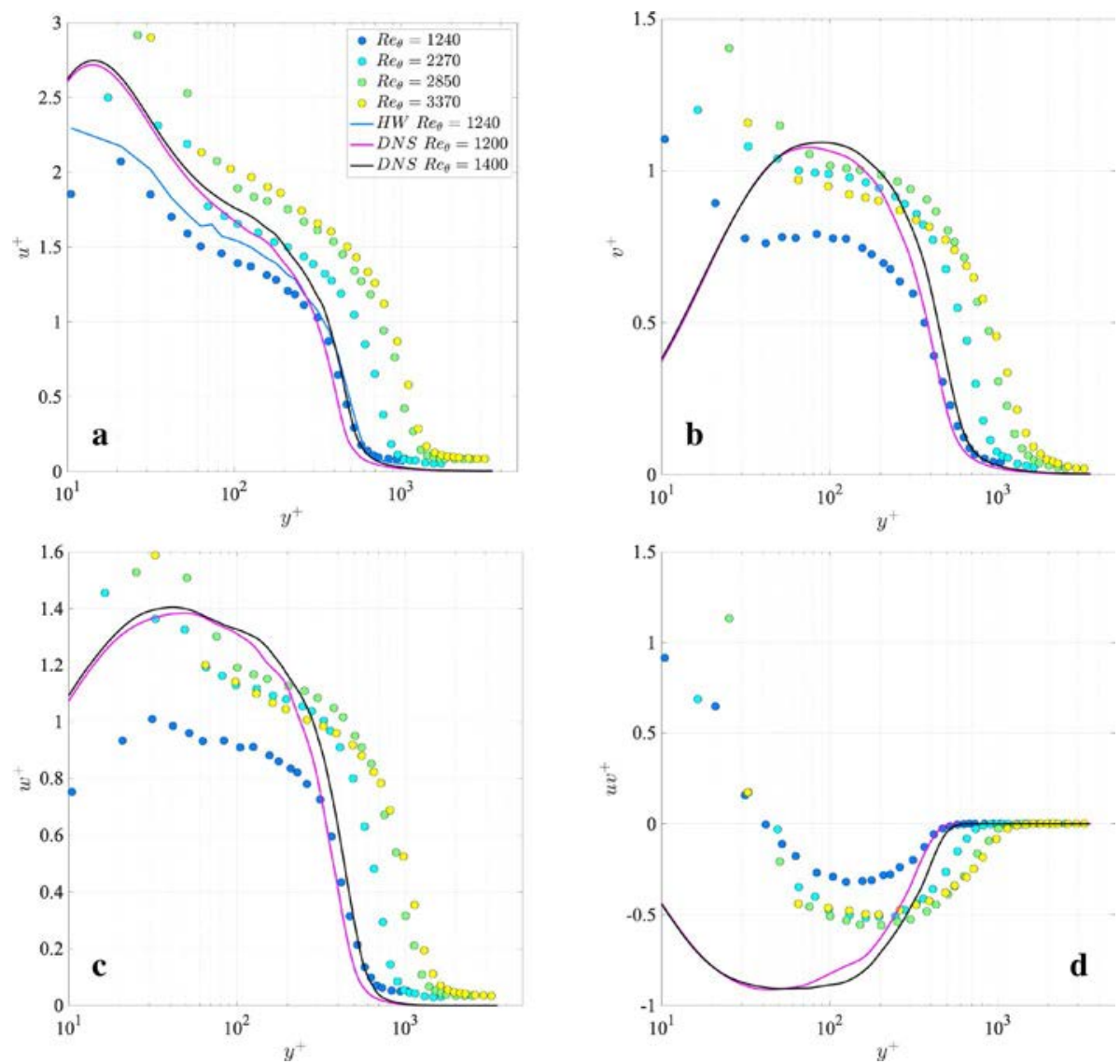

Fig. 4 Normalised fluctuating velocities evolution with $y^{+}$obtained with the Cobra probe (circles), the HW (blue solid line in (a)) and DNS (magenta and black solid lines) 
experimentally, are underestimated by the Cobra probe by a factor 3 . This confirms the necessity of using the DNS to properly estimate both $u_{\tau}$ and $C_{f}$.

We then study the turbulent flow properties for the range $0.4<y / \delta<0.75$, where the mean and rms values of all three velocity components for DNS, HW and Cobra measurements are consistent (all experimental data reported from Fig. 5 onwards corresponds to this range). The turbulent dissipation rate $\varepsilon$ (that in this case corresponds to $\varepsilon_{i s o}$ from Sect. 2) was estimated via the dissipation spectrum of the HW signal. It was calculated as $\varepsilon=\int 15 v k_{1}^{2} E_{11} d k_{1}$, where $E_{11}\left(k_{1}\right)$ is the $1 \mathrm{D}$ power spectrum. As for the DNS, this definition involves assuming local, small-scale, isotropy and homogeneity and in this case, the Taylor hypothesis. The noise at high frequencies has been removed and modelled as a power law, fitted for each time signal [following the protocol from Mora et al. (2019)]. The Taylor micro-scale has been obtained from $\varepsilon$ as $\lambda=\sqrt{15 v u^{\prime 2} / \varepsilon}$. Finally, the integral length scale is defined via the autocorrelation function (Fig. 3b) as $L=\int_{0}^{\rho_{0}} R_{u u}(\rho) d \rho$, where $R_{u u}(\rho)=\langle u(x) u(x+\rho)\rangle / u^{\prime 2}$, and $\rho_{0}$ corresponds to the first zero crossing, $R_{u u}\left(\rho_{0}\right)=0$. The Taylor hypothesis is used here too, to convert from the time autocorrelation (defined in a temporal variable $\tau$ ) to the spatial one as $\rho=U \tau$.

While we already discussed the presence of a $\sim-5 / 3$ power law on the power spectral density for $\operatorname{Re}_{\theta}>1240$, our measurements suggest that the flow remain turbulent, with $R e_{\lambda}>50$ (Fig. 5c). We also see that this parameter varies significantly, a condition to disentangle equilibrium from non-equilibrium turbulence (that has to be complemented with the variation of $\sqrt{R e_{G}} / R e_{\lambda}$, as we will discuss below). Other quantities, such as $\eta, \lambda$ and $\varepsilon$ (Fig. 5a, b and e) also show clear trends with $U_{\infty}, x$ and $y^{+}$. Remarkably, $L$ remains almost constant with these two parameters (5d), remaining at $L \sim 2 \mathrm{~cm}$ for all conditions considered. We also observe that $\eta$ remains always below $350 \mu \mathrm{m}$ therefore 5 times smaller than the Cobra characteristic length (taken as $2 \mathrm{~mm}$, the square root of the sensitive area). This last parameter is also of the same order as $\lambda$, and we therefore confirm that this probe
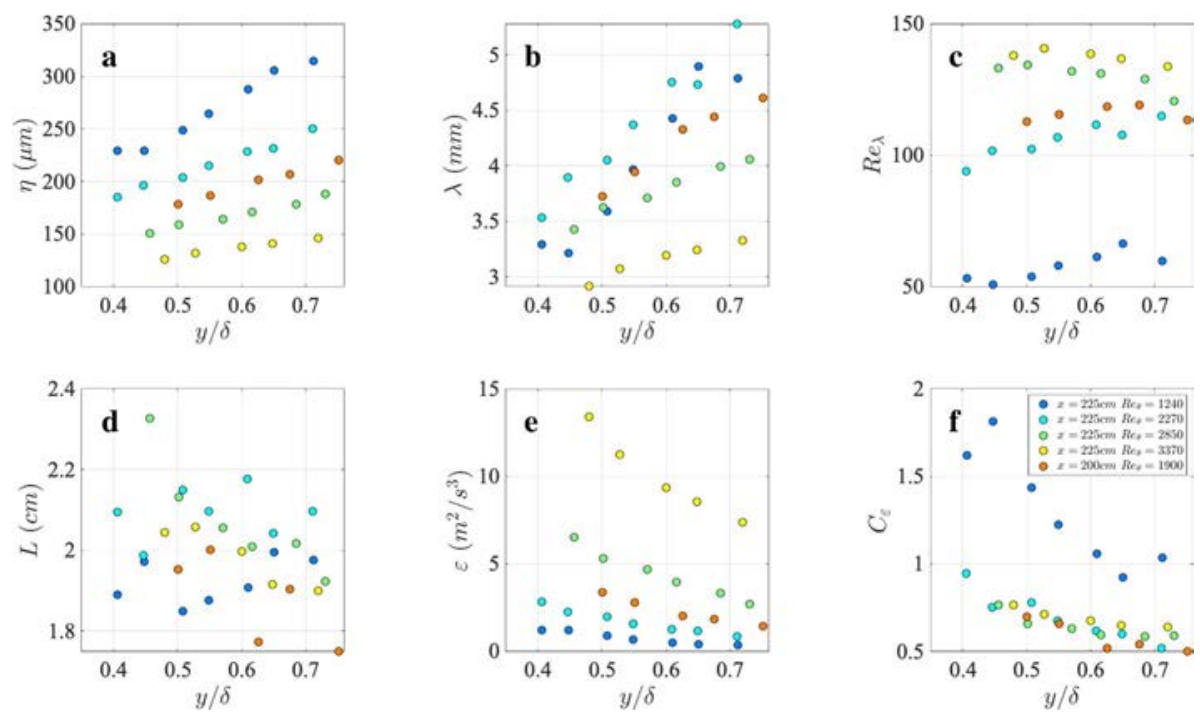

Fig. 5 Turbulence parameters obtained with the HW. Kolmogorov lengthscale (a), Taylor microscale (b), $R e_{\lambda}(\mathbf{c})$, integral lengthscale $L(\mathbf{d})$, turbulent dissipation rate $\varepsilon(\mathbf{e})$ and dissipation constant $C_{\varepsilon}(\mathbf{f})$ 
cannot resolve the small-scale parameters from our experimental setup. We will therefore only use this probe to estimate large-scale averaged quantities such as $(U, V, W)$ and $\left(u^{\prime}, v^{\prime}, w^{\prime}\right)$.

Figure 6 shows a comparison between HW and DNS turbulence parameters, normalised with wall units. As the DNS do not resolve the value of $L, C_{\varepsilon}$ cannot be estimated. Large deviations observed for the DNS for $y^{+}>400$ correspond to $y / \delta>1$ and therefore outside the TBL (Fig. 3a), where the DNS values of $u^{\prime}$ and $\varepsilon$ are close to zero and therefore more affected by numerical noise (Fig. 6b shows that the value of $R e_{\lambda}$ obtained from the DNS still approaches to zero outside the TBL). While parameters present similar values, we can see that the turbulence quantities estimated with the DNS do not match the HW results. This has been indeed reported before, as even DNS of the TBL, within the range of $R e_{\theta}$ studied here, happens to be very sensitive to inflow condition, domain size, etc... (Schlatter and Örlü 2010).

We can then compute $C_{\varepsilon}$ from the HW data from $\varepsilon=C_{\varepsilon} u^{\prime 3} / L$ (Fig. 5f). A very conservative estimation of the relative error of this parameter gives error bars below $20 \%$. The main source of error comes from $\varepsilon$, that can be estimated at around 10\%, considering
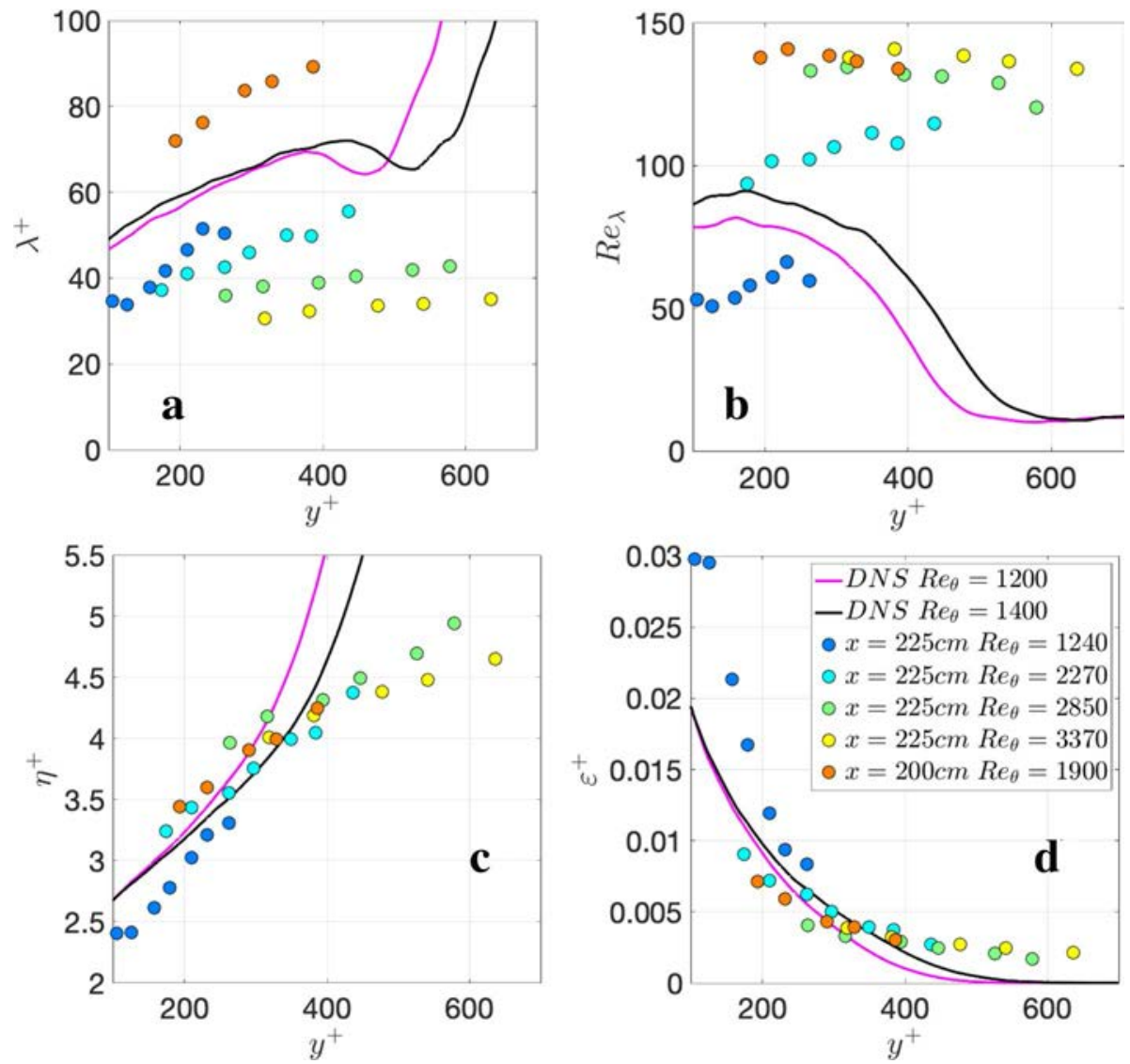

Fig. 6 Turbulence parameters obtained with the HW and DNS: Taylor microscale (a), $\operatorname{Re}_{\lambda}(\mathbf{b})$, Kolmogorov lengthscale (c) and turbulent dissipation rate $\varepsilon(\mathbf{d})$. All dimensional parameters have been normalised with wall units. For a better comparison with the HW, DNS results have been computed using $\varepsilon_{\text {iso }}$ 
the resolution of our wire and the method we use to model the large wavenumbers on the dissipation spectrum, the error of $u^{\prime}$ could be equated to the error of the mean velocity, that is also the main source of error on the estimation of $L$ (due to the use of the Taylor hypothesis).

To identify the properties of the cascade via the dissipation scalings, as detailed in the introduction, we have first to check if $C_{\varepsilon}$ remains constant for varying $R e_{\lambda}$ (Fig. 7a, while we will discuss the role of $R e_{G}$ below). We indeed find the same trends as Nedić et al (the data from Nedić et al. (2017) is also shown in Fig. 7): a constant value of around 0.6 at large $R e_{\lambda}$ but a dependency $C_{\varepsilon} \sim R e_{\lambda}^{-1}$ for low values of $R e_{\theta}$. While our values of $R e_{\theta}$ remain always below the proposed threshold of 10000, we do find $C_{\varepsilon}=c s t$ already at $R e_{\theta} \sim 2500$ (consistently with previous findings on a rough TBL Kamruzzaman et al. 2018). We remind that results at $R e_{\theta}=1240$ do not present a clear $5 / 3$ power law, but they still seem to be in good agreement with previously reported values. Nevertheless, all data from Fig. 7 has a gap on the range $R e_{\lambda} \in$ [150-200], making it difficult to conclude precisely where the transition occurs.

These results are confirmed by the evolution of $\lambda / L$ with $R e_{\lambda}$ (Fig. 7b). While the dependency $\lambda / L \sim R e_{\lambda}^{-1}$ is expected for the Richardson-Kolmogorov cascade, $\lambda / L \sim R e_{G}^{-1 / 2}$ is compatible with non-equilibrium turbulence. Results are consistent with Fig. $7 \mathrm{a}$, as the ratio is constant, but surprisingly no trace of $R e_{G}$ dependency is found in this figures.

We remark that while results from Nedić et al. (2017) are discriminated also by the value of $y / \delta$, due to the large amount of experimental conditions presented here, we decided to plot our results discriminated by this parameter separately on Fig. 9. Our data presents similar trends and values as previous results, like the DNS from Wu et al. (2014): at fixed $R e_{\lambda}$ we see that $C_{\varepsilon}$ slightly decreases for increasing $y / \delta$. At larger values of $R e_{\theta}$, $C_{\varepsilon} \sim 0.6$ seems also to be in good agreement with our data and previously reported values for smooth (Marusic et al. 2015) and rough TBLs (Kamruzzaman et al. 2018).

Nevertheless, there still remains the open question about the influence of the large-scale anisotropy on the estimation of $C_{\varepsilon}$ and the validity of the conclusions stated above. We
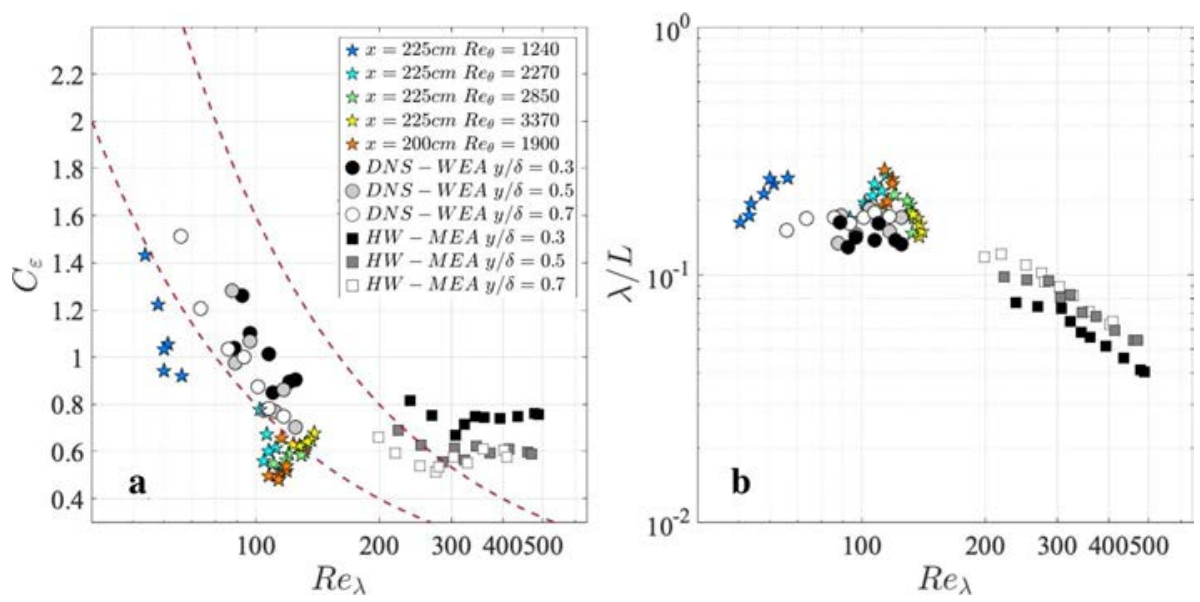

Fig. 7 Comparison of the dependency with $R e_{\lambda}$ of $C_{\varepsilon}$ (a) and $\lambda / L$ (b) obtained via HW in the present experimental setup with the data reported in Nedić et al. (2017) (black and white symbols). Circles correspond to the DNS from Wu et al. (2014) and squares to HW data reported by Marusic et al. (2015). The red dashed lines in (a) are different $R e_{\lambda}^{-1}$ laws for comparison 
have discussed the role of small scale anisotropy in the estimation of $C_{\varepsilon}$ on section 2 . We now show on Fig. $8 \mathrm{a}$ a comparison of the $y^{+}$profiles of $u^{+2}$ and the kinetic energy $K^{+}$, defined as $K^{+}=\frac{1}{2}\left(u^{\prime 2}+v^{\prime 2}+w^{\prime 2}\right) / u_{\tau}^{2}$. Low values of $R e_{\theta}$ have similar trends and values when compared with previous DNS (Pope 2001) at $R e_{\theta}=1410$, as $K^{+} \sim u^{+2}$ in the outer layer. Furthermore, the anisotropy of the flow seems to increase significantly with $R e_{\theta}$. Nevertheless, when the value of $u^{\prime 2}$ or $K$ are used to estimate $C_{\varepsilon}$, the curve remains almost unchanged (Fig. 8b, where both $L$ and $\varepsilon$ are still estimated with the HW, we remind again that the Cobra probe was only used for the value of $K$ on the estimation of $C_{\varepsilon}$ ). We therefore conclude that, for values as large as $R e_{\lambda}=100$ and for $R e_{\theta}<2500$ the classical assumption $C_{\varepsilon}=c s t$ is no longer valid. We remark that we show results using $K$ and not $\frac{2}{3} K$, and therefore the large scales remain anisotropic, but the anisotropy do not modify the trends observed.

In Fig. 9 we further study the behaviour of $C_{\varepsilon}$ and $\lambda / L$. Figures $9 \mathrm{a}-\mathrm{c}$ confirm that our trends with this parameter are consistent with those found by Nedić et al. (2017). In Fig. 9d we study the dependency of $C_{\varepsilon}$ with $\sqrt{R e_{G}} / R e_{\lambda}$. As discussed in the introduction, this relation takes into account not only variations with $R e_{\lambda}$ but it also assesses the role of $R e_{G}$ in the non equilibrium energy cascade. A non-equilibrium energy cascade implies, for the same flow, that $C_{\varepsilon}$ should be a linear function of $\sqrt{R_{G}} / R e_{\lambda}$ (collapsing for all $R e_{G}$ and $R e_{\lambda}$ within this regime). It is not clear how such parameter will be defined in the TBL, and in Fig. $9 \mathrm{~d}$ we use $R e_{G}=\frac{\delta U_{\infty}}{v}$ (and therefore $R e_{G}=R e_{\delta}$ ). As both $\delta$ and $L$ are relatively constant, our definition of $R^{v} e_{G}$ ultimately quantifies variations of $U_{\infty}$, that should be present on all definitions of such parameter. In grid turbulence and some free-shear flows, this parameter remains constant at fix streamwise position $x$ and different $R e_{G}$ (i.e., variations of $\sqrt{R e_{G}}$ and $R e_{\lambda}$ compensate each other at fixed position $x$ when the freestream velocity is changed), and therefore the only way to study the nature of the cascade is via streamwise profiles, where indeed, at fixed value of $U_{\infty}$, the parameter $\sqrt{R e_{G}} / R e_{\lambda}$ changes. Interestingly, this is not the case for the outer region of the TBL, and even profiles at $x=c s t$ present important variations. We have nevertheless left the value of $x$ in Fig. 7 for reference. For comparison, the HW results from the
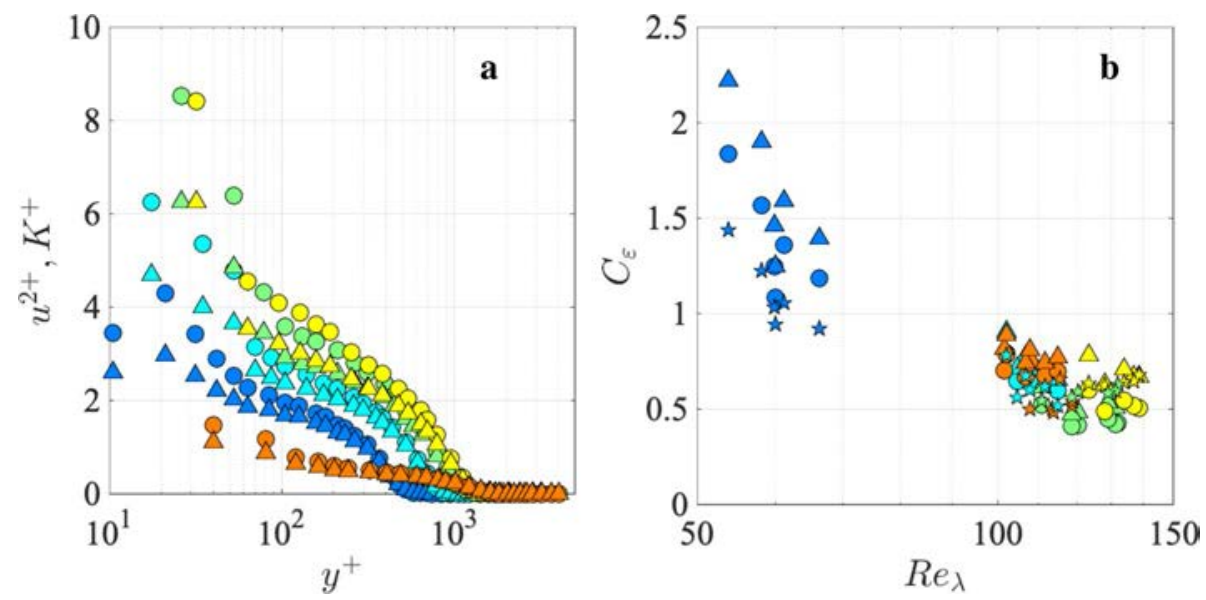

Fig. 8 Comparison between the values of $u^{+2}$ and $K^{+}$(a) obtained with the Cobra probe. Different definitions of $C_{\varepsilon}(\mathbf{b})$. Circles stand for the values of $u^{+2}$ and triangles for $K^{+}$. In figure (b) star markers correspond to HW data. The colours refer to the same datasets as in Fig. 7 

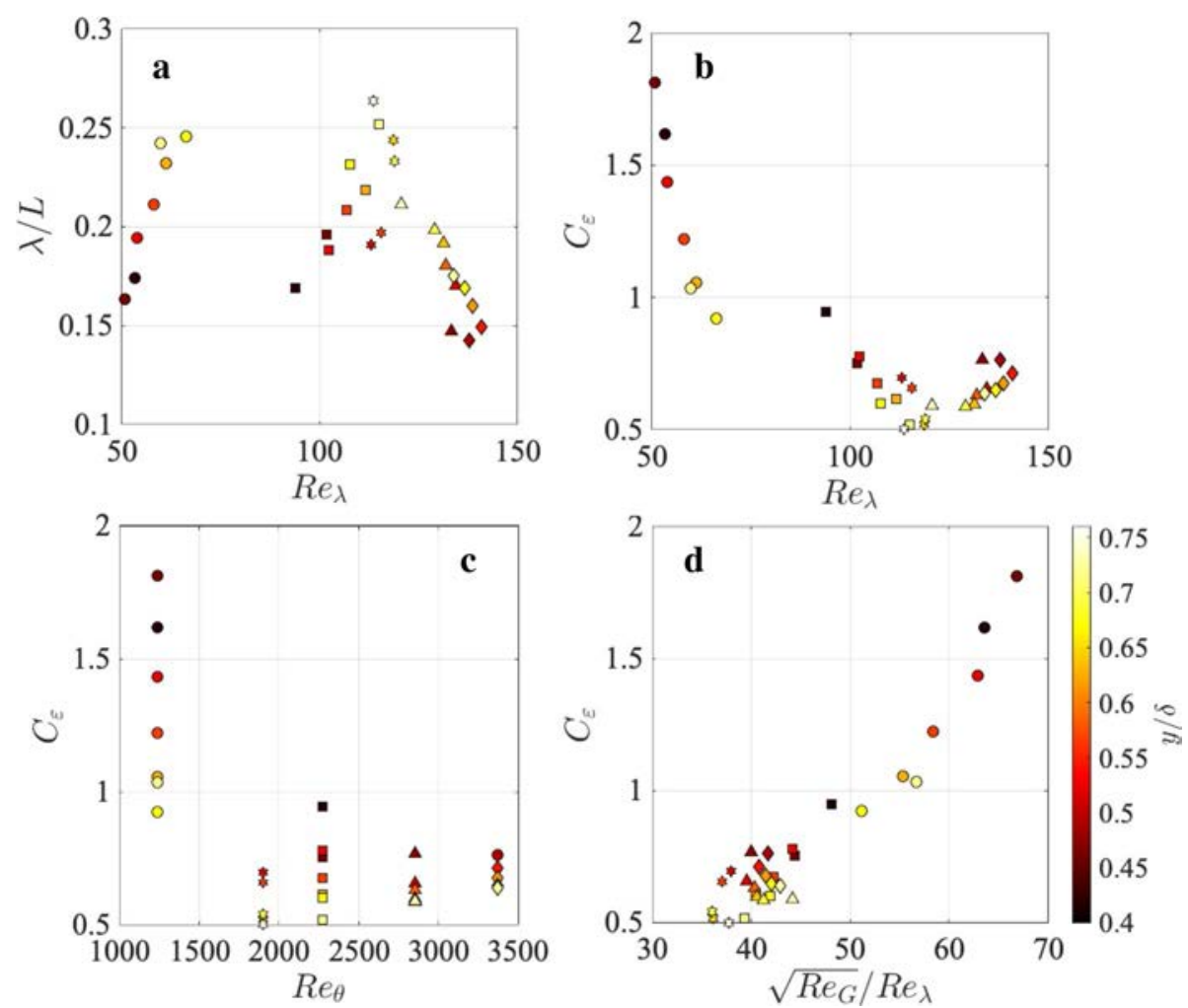

Fig. 9 Different parameters obtained with the HW for our data alone. $\lambda / L$ vs $R e_{\lambda}(\mathbf{a})$. $C_{\varepsilon}$ as a function of $R e_{\lambda}$ (b), $R e_{\theta}(\mathbf{c})$ and $\sqrt{R e_{G}} / R e_{\lambda}(\mathbf{d})$. In all figures the colormap quantifies the position $y / \delta$ where data was taken. Circles stand for $R e_{\theta}=1240$, stars for $R e_{\theta}=1900$, squares for $R e_{\theta}=2300$, triangles for $R e_{\theta}=2900$ and diamonds for $R e_{\theta}=3400$

literature in Fig. 7 (Nedić et al. 2017; Marusic et al. 2015), correspond to a larger wind tunnel at $U_{\infty}=20 \mathrm{~m} / \mathrm{s}$ and values of $\delta$ that range from 45 to $242 \mathrm{~mm}$. For that work, data has been taken at constant $U_{\infty}$ and different values of $x$, and remarkably it results in an almost constant range of $\sqrt{R e_{G}} / R e_{\lambda}$, that ranges from 1.2 up to 1.4. Within that range, and for $y / \delta=0.7$, the authors obtain $C_{\varepsilon} \sim 0.58$, in good agreement with the results from Fig. 9d.

Therefore, a requirement to verify the presence of this energy cascade is that the parameter $\sqrt{R e_{G}} / R e_{\lambda}$ varies throughout our datasets (as it indeed happens). While in Fig. 7 and 8 no trend was easily observed, in Fig. 9 a trend is indeed present, for low values of $R e_{\lambda}$ (resp. large values of $\sqrt{R e_{G}} / R e_{\lambda}$ ), consistent with a linear relation, for all values of $y / \delta$ and $R e_{\theta}$. This result requires further study as, to the authors knowledge, has not been reported before. A larger wind tunnel that allows to explore wider ranges of $\delta, L$ and $R e_{\lambda}$ could allow to better characterise the transition between both cascades and if the definition of $\operatorname{Re}_{G}$ proposed in this work remains valid. Results discussed above from previous experiments at larger values of $\delta$ (Nedić et al. 2017; Marusic et al. 2015) suggest that studies on the nature of dissipation scalings should not only cover different vertical profiles at fixed streamwise positions, but also different values of $U_{\infty}$. While we cannot conclusively confirm the 
presence of a large Reynolds non-equilibrium cascade, the relations observed in Fig. 9 tends to point towards that direction. We therefore confirm the observation reported by Nedić et al. (2017), and the approximation $C_{\varepsilon}=c s t$ is invalid already at $R e_{\theta}<2500$.

\section{Conclusions}

In this work we used hot-wire anemometry and a Cobra probe to estimate both the full kinetic energy and the turbulence energy dissipation rate experimentally on a TBL. A DNS for the lower value of $R e_{\theta}$ was used to quantify the influence of small scale anisotropy and inhomogeneities on the estimation of $\varepsilon$. It also validated the mean and fluctuating velocities profiles obtained experimentally in the range $0.4<y / \delta<0.75$. Our results and the novelty of this work can be summarised as,

- Our combined approach of DNS, HW and Cobra allowed us to complement the advantages of each technique to counteract resolution problems from each one: numerically expensive estimation (thus not achieved in our case) of $L$ and therefore $C_{\varepsilon}$ with the DNS, bad resolution with the Cobra at small scales (impeding the calculation of small-scale quantities such as $\varepsilon, C_{\varepsilon}$ and $\lambda$ ) and absence of 3D information with the HW. We show that the Cobra and HW together are indeed capable of providing information about the energy cascade, while the DNS allowed us to validate our statistics at small $\operatorname{Re}_{\theta}$.

- The DNS also showed that $\varepsilon$ can be estimated using the homogeneous isotropy assumption (3) in the outer layer. While our experimental setup did not allow us to fully resolve the log law region, this confirms that experiments in larger tunnels can indeed obtain all relevant information by combining Cobra and HW probes.

- We confirm evidence discussed previously (Nedić et al. 2017) about the presence of dissipation scalings consistent with non-equilibrium turbulence at small values of $\operatorname{Re}_{\theta}$. In this point the contribution of this work is twofold. First, we show that while the flow presents large-scale anisotropy, it does not affect the trends of $\varepsilon$, when it is estimated as $\varepsilon=C_{\varepsilon} u^{\prime 3} / L$. Second, we not only confirm the presence of the regime $C_{\varepsilon} \sim R e_{\lambda}^{-1}$ at low $R e_{\theta}$, but in our experiment we also changed the global Reynolds number and find that data collapses reasonably well with $\sqrt{R e_{G}} / R e_{\lambda}$. We therefore confirm and expand the validity of the findings from Nedić et al. (2017). While it is beyond the possibilities of our experimental setup, a systematic study on this scaling would require to disentangle the dependency of $C_{\varepsilon}$ with $R e_{G}^{0.5}$ and $R e_{\lambda}$ separately (as done for instance in the grid experiments from Valente and Vassilicos 2012), via experiments at constant $R e_{\lambda}$ and different $R e_{G}$, and vice-versa.

While our results suggest some form of non-equilibrium turbulence is present, and trends reported previously are not contaminated by inhomogeneities and the anisotropy of the flow, further studies are needed to address conclusively the presence of such cascade. Particularly, as this regime occurs at low values of $R e_{\lambda}$, in some cases where the $5 / 3$ power-law is not very clear, it also remains a possibility that some form of viscosity effects are still present. Nevertheless, our results confirm that the standard assumption of the constancy of $C_{\varepsilon}$ is not valid in TBLs with Reynolds numbers as large as $R e_{\lambda}=100$ and $R e_{\theta} \sim 2500$. 
Our results also suggest that the parameter better suited to characterise this transition is $\sqrt{\operatorname{Re}_{G}} / R e_{\lambda}$ and not $R e_{\theta}$.

In this work we have proposed to define $C_{\varepsilon}$ and $R e_{G}$ with $L$ and $\delta$, respectively, but these two parameters do not vary significantly in our dataset. Our aim in this point is to stimulate discussion on the role of these parameters. Nevertheless, as several length scales can be defined in the TBL, further experiments in larger wind tunnels, exploring broader ranges and larger values of $\delta$ and $R e_{\theta}$, would contribute to understand the role and the definition of different length scales in the energy cascade in the TBL. They would also allow to explore the range $R e_{\lambda} \in[100-300]$, and better characterise the transition observed for HW and DNS studied here in terms of $R e_{\lambda}$ and $R e_{\theta}$.

Funding JHS and EBCS thank the founding of Coordenação de Aperfeiçoamento de Pessoal de Nível Superior-Brasil (CAPES) - Finance Code 001. A part of this work was granted access to the HPC resources of IDRIS under the allocation 2019-A0060107567 made by GENCI. This study was funded by Coordenação de Aperfeiçoamento de Pessoal de Nível Superior-Brasil (CAPES)—Finance Code 001. A part of this work was granted access to the HPC resources of IDRIS (Grant Number 2019-A0060107567) made by GENCI.

\section{Declarations}

Conflict of interest The authors declare that they have no conflict of interest.

\section{References}

Antonia, R.A., Djenidi, L., Danaila, L.: Collapse of the turbulent dissipative range on kolmogorov scales. Phys. Fluids 26(4), 045105 (2014)

Cafiero, G., Vassilicos, J.C.: Non-equilibrium turbulence scalings and self-similarity in turbulent planar jets. Proceedings of the Royal Society A 475(2225), 20190038 (2019)

Castillo, Luciano, George, William K.: Similarity analysis for turbulent boundary layer with pressure gradient: outer flow. AIAA J. 39(1), 41-47 (2001)

Dairay, T., Obligado, M., Vassilicos, J.C.: Non-equilibrium scaling laws in axisymmetric turbulent wakes. J. Fluid Mech. 781, 166-195 (2015)

Diaz-Daniel, C., Laizet, S., Vassilicos, J.C.: Wall shear stress fluctuations: Mixed scaling and their effects on velocity fluctuations in a turbulent boundary layer. Phys. Fluids 29(5), 055102 (2017)

George, William K., Castillo, Luciano: Zero-pressure-gradient turbulent boundary layer. Appl. Mech. Rev. 50(12), 689-729 (1997)

Goto, S., Vassilicos, J.C.: Local equilibrium hypothesis and Taylor's dissipation law. Fluid Dyn. Res. 48(2), 021402 (2016)

Goto, S., Vassilicos, J.C.: Unsteady turbulence cascades. Phys. Rev. E 94(5), 053108 (2016)

Hearst, Jason: Lavoie, Philippe: effects of multi-scale and regular grid geometries on decaying turbulence. J. Fluid Mech. 803, 528-555 (2016)

Hultmark, M., Vallikivi, M., Bailey, S.C.C., Smits, A.J.: Turbulent pipe flow at extreme Reynolds numbers. Phys. Rev. Lett. 108(9), 094501 (2012)

Kamruzzaman, M.D., Djenidi, L., Antonia, R.A.: Behaviour of the energy dissipation coefficient in a rough wall turbulent boundary layer. Exp. Fluids 59(1), 9 (2018)

Laizet, S., Lamballais, E.: High-order compact schemes for incompressible flows: a simple and efficient method with the quasi-spectral accuracy. J. Comp. Phys. 228(15), 5989-6015 (2009)

Larssen, Jon V., Devenport, William J.: On the generation of large-scale homogeneous turbulence. Exp. Fluids 50(5), 1207-1223 (2011)

Lesieur, Marcel.: Turbulence in fluids, volume 40. Springer Science \& Business Media, (2012)

$\mathrm{Li}$, N., Laizet, S.: Incompact3d, a powerful tool to tackle turbulence problems with up to $0\left(10^{5}\right)$ computational cores. J. Num. Methods Fluids 67(11), 1735-1757 (2011) 
Liu, F., Fang, L., Fang, J.: Non-equilibrium turbulent phenomena in transitional flat plate boundary-layer flows. Appl. Math. Mech. 42(4), 567-582 (2021)

Lumley, J.L.: Some comments on turbulence. Phys. Fluids A Fluid Dyn. 4(2), 203-211 (1992)

Marusic, I., Chauhan, K.A., Kulandaivelu, V., Hutchins, N.: Evolution of zero-pressure-gradient boundary layers from different tripping conditions. J. Fluid Mech. 783, 379-411 (2015)

Marusic, I., Hutchins, N., Mathis, R.: High Reynolds number effects in wall turbulence. In TSFP DIGITAL LIBRARY ONLINE. Begel House Inc., (2009)

Mi, J., Antonia, R.A.: Approach to local axisymmetry in a turbulent cylinder wake. Exp. Fluids 48(6), 933947 (2010)

Monin, Andre Sergeevich., Yaglom, Akiva M.: Statistical fluid mechanics, volume II: mechanics of turbulence, volume 2 Courier Corporation, (2013)

Mora, D.O., Pladellorens, E.M., Turró, P.R., Lagauzere, M., Obligado, M.: Energy cascades in active-gridgenerated turbulent flows. Phys. Rev. Fluids 4(10), 104601 (2019)

Nagata, K., Saiki, T., Sakai, Y., Ito, Y., Iwano, K.: Effects of grid geometry on non-equilibrium dissipation in grid turbulence. Phys. Fluids 29(1), 015102 (2017)

Nedić, J., Tavoularis, S., Marusic, I.: Dissipation scaling in constant-pressure turbulent boundary layers. Phys. Rev. Fluids 2(3), 032601 (2017)

Nedić, J., Tavoularis, S.: Energy dissipation scaling in uniformly sheared turbulence. Phys. Rev. E 93(3), 033115 (2016)

Obligado, M., Dairay, T., Vassilicos, J.C.: Nonequilibrium scalings of turbulent wakes. Phys. Rev. Fluids 1(4), 044409 (2016)

Phillips, W.R.C., Ratnanather, J.T.: The outer region of a turbulent boundary layer. Phy. Fluids A Fluid Dyn. 2(3), 427-434 (1990)

Pope, SB.: Turbulent flows, (2001)

Pumir, A., Xu, H., Siggia, E.D.: Small-scale anisotropy in turbulent boundary layers. J. Fluid Mech. 804, 5 (2016)

Schlatter, P., Örlü, R.: Assessment of direct numerical simulation data of turbulent boundary layers. J Fluid Mech. 659, 116-126 (2010)

Schlatter, P., Örlü, R.: Turbulent boundary layers at moderate Reynolds numbers: inflow length and tripping effects. J. Fluid Mech. 710, 5 (2012)

Schlatter, P., Li, Q., Brethouwer, G., Johansson, A.V., Henningson, D.S.: Simulations of spatially evolving turbulent boundary layers up to $\operatorname{re} \theta=4300$. Int. J. Heat Fluid Flow 31(3), 251-261 (2010)

Smits, A.J., McKeon, B.J., Marusic, I.: High-reynolds number wall turbulence. Annu. Rev. Fluid Mech. 43, 353-375 (2011)

Solak, Ilkay, Laval, Jean-Philippe.: Large-scale motions from a direct numerical simulation of a turbulent boundary layer. Phys. Rev. E 98(3), 033101 (2018)

Sreenivasan, KR.: The turbulent boundary layer. In Frontiers in experimental fluid mechanics, pages 159209. Springer, (1989)

Takamure, K., Sakai, Y., Ito, Y., Iwano, K., Hayase, T.: Dissipation scaling in the transition region of turbulent mixing layer. Int. J. Heat Fluid Flow 75, 77-85 (2019)

Valente, P.C., Onishi, R., da Silva, C.B.: Origin of the imbalance between energy cascade and dissipation in turbulence. Phys. Rev. E 90(2), 023003 (2014)

Valente, P.C., Vassilicos, J.C.: Universal dissipation scaling for nonequilibrium turbulence. Phys. Rev. Lett. $\mathbf{1 0 8}(21), 214503$ (2012)

Vassilicos, J.C.: Dissipation in turbulent flows. Annu. Rev. Fluid Mech. 47, 95-114 (2015)

Vassilicos, J.C., Laval, J.-P., Foucaut, J.-M., Stanislas, M.: The streamwise turbulence intensity in the intermediate layer of turbulent pipe flow. J. Fluid Mech. 774, 324-341 (2015)

Watkins, S., Mousley, P., Hooper, J.: Measurement of fluctuating flows using multi-hole probes. In Ninth International Congress on Sound and Vibration, pages 8-11, (2002)

Wu, X., Moin, P., Hickey, J.-P.: Boundary layer bypass transition. Phys. Fluids 26(9), 091104 (2014) 